\title{
Germinação e Crescimento Inicial Entre Matrizes de Duas Espécies do Gênero Hymenaea
}

\author{
Priscila Fernandes de Souza ${ }^{1}$, Reynaldo Campos Santana ${ }^{1}$, \\ José Sebastião Cunha Fernandes ${ }^{2}$, Luiz Felipe Ramalho de Oliveira ${ }^{1}$, \\ Evandro Luiz Mendonça Machado ${ }^{1}$, Marcela Carlota Nery², \\ Marcio Leles Romarco de Oliveira ${ }^{1}$
}

${ }^{1}$ Departamento de Engenharia Florestal, Universidade Federal dos Vales do Jequitinhonha e Mucuri - UFVJM, Diamantina/MG, Brasil

${ }^{2}$ Departamento de Agronomia, Universidade Federal dos Vales do Jequitinhonha e Mucuri - UFVJM, Diamantina/MG, Brasil

\begin{abstract}
RESUMO
O gênero Hymenaea pertence à família Leguminosae, subfamília Caesalpinoideae, com nome popular de jatobá. No gênero Hymenaea, foram descritas aproximadamente 25 espécies. Já no Brasil, verifica-se a presença de 13 espécies, com destaque para a Hymenaea martiana Hayne e a Hymenaea courbaril Linneaus. Na literatura, há vários trabalhos que tratam principalmente da quebra de dormência das sementes e da produção de mudas, sobretudo da espécie Hymenaea courbaril Linneaus, porém essas avaliações por matriz ainda são incipientes. Este trabalho teve por objetivo avaliar e comparar a germinação e o crescimento inicial de mudas entre as espécies e as matrizes de Hymenaea martiana Hayne e Hymenaea courbaril Linneaus. Foram desenvolvidos dois experimentos: o primeiro avaliou a germinação, e o segundo, o crescimento inicial de mudas. As duas espécies avaliadas apresentaram comportamentos distintos entre a germinação e o crescimento inicial das mudas, sendo a Hymenaea courbaril Linneaus a espécie com maior média para ambas variáveis, porém não apresentou variabilidade fenotípica entre matrizes.
\end{abstract}

Palavras-chave: jatobá, variabilidade fenotípica, produção de mudas.

\section{Germination and Seedling Growth Among Matrices of Two Species of the Genus Hymenaea}

\begin{abstract}
The Hymenaea genus belongs to the family Leguminosae, subfamily Caesalpinoideae with the common name jatobá. Approximately 25 species have been described in the genus Hymenaea and 13 of them are present in Brazil. Among them we can highlight the species Hymenaea martiana Hayne and Hymenaea courbaril Linneaus. In literature, there are several studies dealing mainly the dormancy breakage and the seedling production, mainly with Hymenaea coubaril Linneaus, but these reviews per matrices are incipient. This study aimed to evaluate and compare the germination and seedling growth between species and between matrices of the Hymenaea martiana Hayne and Hymenaea courbaril Linneaus. Two experiments were conducted: the first evaluated the germination and the second the seedlings growth. The two species studied showed different behavior between germination and the seedling growth, with Hymenaea courbaril Linneaus showing the greatest average for both variables. However, this did not show phenotypic variability between matrices.
\end{abstract}

Keywords: jatobá, phenotypic variability, seedling production. 


\section{INTRODUÇÃO}

O gênero Hymenaea pertence à família Leguminosae, subfamília Caesalpinoideae, com nome popular de jatobá. Esse gênero ocorre amplamente no Brasil e na América tropical (Rizzini, 2000).

O jatobá é uma árvore grande, com 15 a $20 \mathrm{~m}$ de altura (Lorenzi, 2002). Seus frutos comestíveis possuem elevado teor de fibra alimentar, cálcio e magnésio e são utilizados na alimentação humana e animal (Batista et al., 2011). Também é amplamente estudado devido à presença de terpenos, oligossacarídeos e polissacarídeos nas sementes, folhas e cotilédones (Vargas-Rechia et al., 1998; Nogueira et al., 2001; Busato et al., 2001; Santos \& Buckeridge, 2004; Brandão et al., 2009), substâncias essas que apresentam atividade antimicrobiana (Fernandes et al., 2005; Gonçalves et al., 2005).

$\mathrm{O}$ gênero possui flores brancas ou avermelhadas hermafroditas, sendo a polinização realizada por morcegos (Lorenzi, 2002; Silva, 2006). A propagação ocorre basicamente via seminífera e, por possuir dormência tegumentar, a germinação é baixa quando não são utilizados métodos de quebra dormência (Silva, 2006).

A semeadura do jatobá pode ser realizada em sacos de polietileno ou diretamente em campo, sendo que o início da germinação de sementes com dormência superada ocorre de 12 a 60 dias após a semeadura. Esta, ao contrário, pode se prolongar por até 10 meses, e, após seu término, as mudas estão aptas para plantio em cerca de três meses (Carvalho, 2003).

No gênero Hymenaea, foram descritas aproximadamente 25 espécies, a maioria com madeira de alto valor comercial. No Brasil, verifica-se a presença de 13 espécies (Rizzini, 2000), com destaque para a Hymenaea martiana Hayne e a Hymenaea courbaril Linneaus, que são morfologicamente semelhantes, mas diferenciam-se pela presença de pubescência nas folhas e pelo tronco rugoso na primeira (Lorenzi, 2002). Na literatura, são mais frequentes trabalhos que abordam a quebra de dormência das sementes e a produção de mudas, principalmente da espécie Hymenaea courbaril Linneaus, porém avaliações por matriz ainda são incipientes.

Tais informações são importantes, pois há indícios que indivíduos da mesma espécie apresentam padrões variados quanto à germinação, dormência e vigor das plântulas (Machado et al., 2004). Tais fatores genéticos podem ser explorados nos programas de melhoramento (Machado et al., 2004), e, além disso, ajudam a entender as estratégias reprodutivas das espécies (Baskin \& Baskin, 2001) e subsidiar trabalhos de regeneração, silvicultura, conservação e utilização de recursos genéticos (Cruz \& Carvalho, 2003).

Dentro desse contexto, este trabalho teve por objetivo avaliar e comparar a germinação e o crescimento inicial de mudas entre as espécies e as matrizes de Hymenaea martiana Hayne e Hymenaea courbaril Linneaus.

\section{MATERIAL E MÉTODOS}

Os experimentos avaliaram a germinação e o crescimento inicial das mudas de cinco matrizes de Hymenaea courbaril Linneaus, denominadas neste trabalho como M1, M2, M3, M4 e M5, localizadas na Fazenda Experimental do Moura, pertencente à Universidade Federal dos Vales do Jequitinhonha e Mucuri (UFVJM), em uma área contínua de 571 ha, no município de Curvelo, no Estado de Minas Gerais, e de sete matrizes de Hymenaea martiana Hayne, também denominadas de M1, M2, M3, M4, M5, M6 e M7, localizadas em Mendanha, distrito de Diamantina, em Minas Gerais, dentro do perímetro urbano em áreas antropizadas. A coleta dos frutos das duas matrizes ocorreu em agosto de 2010.

As matrizes forma escolhidas aletoriamente, utilizando-se como critério de seleção as com sanidade fitossanitária. Padronizou-se uma distância mínima entre matrizes de $30 \mathrm{~m}$, de forma a impossibilitar a sobreposição das copas, visto que os frutos foram coletados diretamente do chão.

Todos os experimentos foram analisados considerando-se os efeitos entre matrizes e dentro de espécies, com a utilização do software Statistica 10.0 (Statsoft, 2010).

A seguir é apresentada a descrição dos dois experimentos.

\subsection{Experimento 1: germinação}

As sementes foram obtidas por meio da quebra da casca dos frutos com o martelo e separadas do endocarpo com uso de faca. Em seguida, elas foram armazenadas por 90 dias em câmara fria a $6^{\circ} \mathrm{C}$ e umidade relativa 
de $25 \%$ no Centro Integrado de Pesquisa e Estudos Florestais (CIPEF) da UFVJM, em Diamantina-MG.

O grau de umidade das sementes (\%) foi determinado pelo método da estufa a $105^{\circ} \mathrm{C} \pm 3{ }^{\circ} \mathrm{C}$ por 24 horas (Brasil, 2009). As sementes foram quebradas em quatro partes (Andrade et al., 2010), com a ajuda de um martelo, antes de se realizar o teste de umidade. De posse dos dados, calculou-se o grau de umidade (\%).

Antes de realizar o teste de germinação, as sementes foram desinfestadas em solução de cloro ativo a 2,5\% por 5 minutos, escarificadas lateralmente em esmeril e colocadas por 24 horas em água destilada para embebição. Após esse período, foram semeadas em bandejas sob areia de textura média, lavadas e esterilizadas em estufa a $200{ }^{\circ} \mathrm{C}$ por 2 horas (Brasil, 2009) e colocadas para germinar em casa de vegetação.

Diariamente realizou-se o umedecimento manual da areia, atentando-se para que esta não ficasse encharcada, e a contagem das sementes, germinadas. Considerou-se como germinadas as sementes com protrusão radicular superior a $2 \mathrm{~mm}$ de comprimento.

Os resultados foram expressos em porcentagem de sementes germinadas e calculou-se o índice de velocidade de germinação (IVG) de acordo com Maguire (1962).

\subsection{Experimento 2: crescimento inicial das mudas}

Concomitantemente ao teste de germinação, as primeiras plântulas normais desenvolvidas por tratamento foram transplantadas para se avaliar o crescimento inicial das mudas em casa de sombra (50\% de sombreamento) no CIPEF, preservando-se os mesmos tratamentos do experimento de germinação. Aos 30 e 60 dias após o último transplante, foram mensuradas a sobrevivência altura e o diâmetro do coleto das mudas.

As plântulas foram transplantadas para tubetes de $280 \mathrm{~cm}^{3}$ com substrato composto por $70 \%$ vermiculita e $30 \%$ de casca arroz carbonizada. Foi utilizado, para cada 200 litros de substrato, adubo com a formulação: 400 g Osmocote 19:06:10 Mini Prill, 3-4 meses; 1,6 kg supersimples; $1,5 \mathrm{~kg}$ sulfato de amônio; $0,45 \mathrm{~kg}$ cloreto de potássio; $30 \mathrm{~g}$ sulfato de zinco*; $30 \mathrm{~g}$ sulfato de cobre $^{\star} ; 30$ g sulfato de manganês*; 60 g ácido bórico*
( ${ }^{*}$ Diluído em 200 L de água e utilizado 20 L da solução para cada 200 L de substrato).

\subsection{Análise estatística}

O delineamento utilizado nos dois experimentos foi o inteiramente casualizado. Todos os dados obtidos foram submetidos à análise de variância, e as médias comparadas, pelo Tukey a 5\% de probabilidade.

Para a determinação do grau de umidade, utilizaram-se três repetições com cinco sementes por matriz.

O teste de germinação foi instalado com 12 tratamentos (cinco matrizes de Hymenaea courbaril Linneaus e sete matrizes de Hymenaea martiana Hayne) e quatro repetições contendo 25 sementes por parcela para cada matriz, totalizando 100 sementes. Além disso, a germinação acumulada foi obtida ao se ajustar o modelo logístico $\mathrm{y}=\mathrm{a} /(1+\mathrm{b}$ e $(-\mathrm{cx}))(1)$ e os comportamentos foram comparados pelo teste de identidade de modelo não lineares, segundo Souza et al. (2013a).

Com relação ao crescimento inicial das mudas, foram avaliados os mesmos tratamentos da germinação (12 tratamentos), com quatro repetições e 20 mudas por parcela. Os dados de sobrevivência, altura e diâmetro foram analisados pela média da parcela e o arranjo utilizado foi o de parcelas subdivididas. Já o tratamento principal (parcela) foi analisado pelas matrizes, e o subtratamento (subparcela), pelo tempo.

\section{RESULTADOS E DISCUSSÃO}

\subsection{Hymenaea martiana Hayne}

A espécie Hymenaea martiana Hayne apresentou um grau de umidade de $14,09 \%$, valor pouco acima do encontrado por Cruz et al. (2001) para sementes de Hymenaea intermedia Ducke.

Para as variáveis germinação e IVG, foram encontradas diferenças significativas entre matrizes dentro da espécie (Tabela 1), sendo a média geral dessas variaríeis, respectivamente, de 66\% e 0,94 (Tabela 2). O resultado da germinação foi inferior ao de $96 \%$ obtido para a Hymenaea intermedia Ducke (Cruz et al., 2001). As matrizes de Hymenaea martiana Hayne que apresentaram maior média para germinação foram M4, M5 e M6 e para o IVG, foram M4 e M6, todas com germinação maior ou igual a $80 \%$ (Tabela 2 ). 
Uma abordagem mais precisa pode ser feita com a utilização das equações ajustadas e por meio do teste de identidade de modelos não lineares, pois uma avaliação conjunta da maior taxa de germinação e um percentual final de germinação são importantes para a detecção inicial dos melhores materiais genéticos (Costa et al., 2005). Com essa análise concluiu-se que as matrizes possuem comportamentos germinativos diferentes (Figura 1). Para a espécie Hymenaea martiana Hayne, a matriz que apresentou tais características foi a M5. Esses resultados também foram obtidos na seleção de

Tabela 1. Resumo da análise de variância referente às variáveis porcentagem de germinação $(G)$ e índice de velocidade de germinação (IVG) de sete matrizes de Hymenaea martiana Hayne (Esp 1) e cinco matrizes de Hymenaea courbaril Linneaus (Esp 2). Diamantina-MG, novembro de 2010.

Table 1. Resume of variance analyses to the variables percentage of germination (G) and IVG of seven matrices of species Hymenaea martiana Hayne (Esp 1) and five matrices of Hymenaea courbaril Linneaus (Esp 2). Diamantina-MG, november 2010.

\begin{tabular}{|c|c|c|c|}
\hline \multirow{2}{*}{ FV } & \multirow{2}{*}{ GL } & G & IVG \\
\hline & & QM & QM \\
\hline Esp e Matrizes & 11 & $1301,4^{*}$ & $0,31^{*}$ \\
\hline Entre Espécies & 1 & $803,7^{\star}$ & $0,02^{\mathrm{ns}}$ \\
\hline Matriz/Espécie & 10 & $1351,1^{*}$ & $0,34^{*}$ \\
\hline Matriz/Esp 1 & 6 & $2013,3^{*}$ & $0,51^{*}$ \\
\hline Matriz/Esp 2 & 4 & $357,8^{\star}$ & $0,10^{*}$ \\
\hline Rep/Matriz & 36 & 133,4 & 0,03 \\
\hline Total & 47 & & \\
\hline CV (\%) & & 16,6 & 18,2 \\
\hline
\end{tabular}

FV: fonte de variação; GL: grau de liberdade; QM: quadrado médio; Rep.: repetição; Esp: espécie. ns: não significativo. ${ }^{*}$ significativo a $5 \%$ pelo teste $t$. matrizes potencias para a germinação de sementes de Albizia lebbeck (L.) Benth e Hevea brasiliensis (Willd. ex Adr. de Juss.) Muell-Arg. (Rego et al., 2005; Costa et al., 2005).

Houve diferenças significativas entre as matrizes para as variáveis altura, diâmetro e sobrevivência (Tabela 3). Porém somente aos 60 dias houve diferenças significativas da altura das mudas entre matrizes, sendo a matriz M5 a de maior média (Tabela 4). Com relação ao diâmetro, as matrizes M4 e M5 foram a de maior média (Tabela 5). A média geral para as variáveis altura, diâmetro e sobrevivência, foram, respectivamente, aos 30 dias, $6,65 \mathrm{~cm}, 3,43 \mathrm{~mm}$ e 57,30\% e, aos 60 dias, $12,16 \mathrm{~cm}, 3,44 \mathrm{~mm}$ e $53,54 \%$.

Quanto à sobrevivência, a matriz M3 não poderia ser selecionada, pois apresentou $0 \%$ de sobrevivência (Tabela 5), o que demostra a importância do conhecimento prévio do comportamento do crescimento de mudas de matrizes antes da implantação de um programa de melhoramento. Além disso, também apresentou o pior comportamento com relação a germinação, com uma reduzida taxa e porcentagem final de germinação (Figura 1).

\subsection{Hymenaea courbaril Linneaus}

A espécie Hymenaea courbaril Linneaus apresentou um grau de umidade de $14,19 \%$, valor semelhante ao encontrado por Souza et al. (2013b) ao armazenar sementes de Hymenaea courbaril Linneaus por até seis meses em freezer.

Para as variáveis germinação e IVG, foram encontradas diferenças significativas entre matrizes dentro da espécie (Tabela 1), porém não houve diferenças entre as

Tabela 2. Valores médios das características porcentagem de germinação $(\mathrm{G})$ e índice de velocidade de germinação (IVG) de sete matrizes de Hymenaea martiana Hayne e cinco matrizes de Hymenaea courbaril Linneaus. Diamantina-MG, novembro de 2010.

Table 2. Medium values of characteristics percentage of germination (G) and IVG of seven matrices of species Hymenaea martiana Hayne and five matrices of Hymenaea courbaril Linneaus. Diamantina-MG, november 2010.

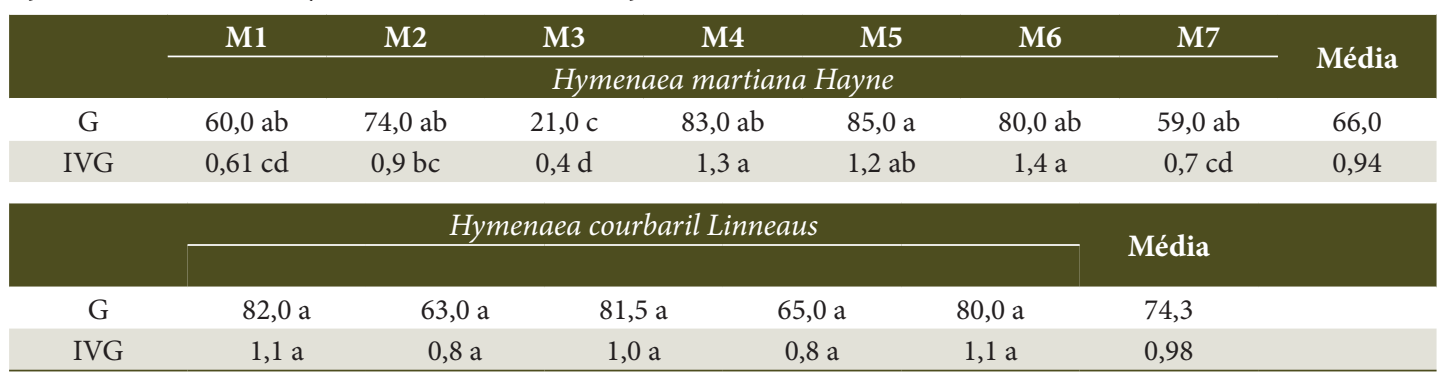

Médias seguidas pela mesma letra na linha não diferem entre si, pelo teste Tukey, a 5\% de probabilidade; M: matriz. 
Tabela 3. Resumo da análise de variância referente às variáveis altura (A), diâmetro (D) e sobrevivência (S) das mudas de sete matrizes de Hymenaea martiana Hayne (Esp 1) e cinco matrizes de Hymenaea courbaril Linneaus (Esp 2). Diamantina-MG, novembro de 2010.

Table 3. Resume of variance analyses to the variables height (A), diameter (D) and survival (S) seedlings of seven matrices of species Hymenaea martiana Hayne (Esp 1) and five matrices of Hymenaea courbaril Linneaus (Esp 2). Diamantina-MG, november 2010.

\begin{tabular}{|c|c|c|c|c|}
\hline & & A & D & $\mathbf{S}$ \\
\hline FV & GL & QM & QM & QM \\
\hline Esp e Mat & 11 & $122,3^{*}$ & $11,0^{*}$ & $4467,7^{\star}$ \\
\hline Esp & 1 & $119,1^{*}$ & $18,7^{\star}$ & $7108,2^{*}$ \\
\hline Mat/Esp & 10 & $122,61^{*}$ & $10,2^{*}$ & $4203,6^{*}$ \\
\hline Mat/Esp1 & 6 & $203,0^{*}$ & $16,9^{*}$ & $6403,3^{*}$ \\
\hline Mat/Esp2 & 4 & $1,93^{\text {ns }}$ & $0,2^{\text {ns }}$ & $904,1^{\text {ns }}$ \\
\hline Resíduo (a) & 36 & 80,9 & 6,0 & 3465,4 \\
\hline $\mathrm{CVa}(\%)$ & & 46,9 & 25,8 & 64,2 \\
\hline Tempo & 1 & $1036,3^{*}$ & $0,2^{\text {ns }}$ & $2830,4^{\text {ns }}$ \\
\hline Tempo x Esp e Mat & 11 & $31,1^{\star}$ & $0,6^{\mathrm{ns}}$ & $699,6^{\mathrm{ns}}$ \\
\hline Resíduo (b) & 36 & 9,9 & 0,5 & 427,4 \\
\hline CVb (\%) & & 30,4 & 20,9 & 55,1 \\
\hline Total & 95 & 1820,8 & 93,4 & 32063,9 \\
\hline
\end{tabular}

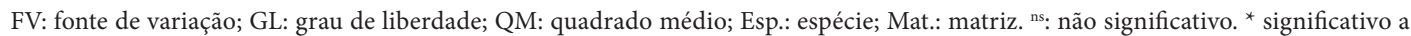
$5 \%$ pelo teste $\mathrm{t}$.

Tabela 4. Valores médios da altura de mudas de sete matrizes de Hymenaea martiana Hayne e cinco matrizes de Hymenaea courbaril Linneaus em dois tempos (30 e 60 dias). Diamantina-MG, novembro de 2010.

Table 4. Medium values of the height of seedlings of seven matrices of species Hymenaea martiana Hayne and five matrices of Hymenaea courbaril Linneaus in two times (30 and 60 days). Diamantina-MG, november 2010.

\section{Matriz}

\begin{tabular}{clccc} 
& 30 & 60 & 30 & 60 \\
\hline M1 & Hymenaea martiana & Hayne & Hymenaea courbaril Linneaus \\
M2 & $6,9 \mathrm{Aa}$ & $8,3 \mathrm{Acd}$ & $8,2 \mathrm{Ba}$ & $14,9 \mathrm{Aa}$ \\
M3 & $7,5 \mathrm{Aa}$ & $11,5 \mathrm{Abc}$ & $5,4 \mathrm{Ba}$ & $17,7 \mathrm{Aa}$ \\
M4 & $0 \mathrm{Aa}$ & $0 \mathrm{Ad}$ & $8,7 \mathrm{Ba}$ & $15,7 \mathrm{Aa}$ \\
M5 & $9,4 \mathrm{Ba}$ & $18,5 \mathrm{Aab}$ & $7,7 \mathrm{Ba}$ & $14,2 \mathrm{Aa}$ \\
M6 & $8,3 \mathrm{Ba}$ & $21,9 \mathrm{Aa}$ & $8,1 \mathrm{Ba}$ & $15,9 \mathrm{Aa}$ \\
M7 & $8,7 \mathrm{Ba}$ & $14,6 \mathrm{Aabc}$ & & 15,69 \\
\hline
\end{tabular}

Médias seguidas por mesma letra, maiúscula na linha e minúscula na coluna, dentro de uma mesma espécie, não diferem estatisticamente entre si pelo teste de Tukey, a 5\% de probabilidade; M: matriz.

médias das matrizes pelo teste de Tukey. A média geral para essas variáveis foram, respectivamente, de $74,3 \%$ e 0,98 (Tabela 2). Em um experimento semelhante a este, com a mesma espécie, o percentual foi de $60 \%$, sendo inferior ao encontrado (Azeredo et al., 2003).

Assim como ocorreu para a espécie Hymenaea martiana Hayne, o teste de identidade de modelos não lineares proporcionou uma análise mais precisa da germinação, demostrando que todas as matrizes dentro de cada espécie Hymenaea courbaril Linneaus apresentaram comportamento germinativo estatisticamente diferentes (Figura 2). A matriz que apresentou maior taxa de germinação e percentual final de germinação foi a M3 (Figura 2).

Não houve diferenças significativas entre as matrizes para as variáveis altura, diâmetro e sobrevivência (Tabela 3), sendo que aos 30 e 60 dias não houve diferenças entre as médias de altura das mudas das 
matrizes (Tabela 4). A não diferença encontrada entre matrizes para o crescimento (altura e diâmetro) pode ser pelo fato de o desenvolvimento inicial ser apoiado, principalmente, pela mobilização do xiloglucano de

Tabela 5. Valores médios do diâmetro e sobrevivência de mudas de sete matrizes de Hymenaea martiana Hayne. Diamantina-MG, novembro de 2010.

Table 5. Medium values of the diameter and survival of seedlings of different matrices of Hymenaea martiana Hayne. Diamantina-MG, november 2010.

\begin{tabular}{|ccc|}
\hline Matriz & Diâmetro & Sobrevivência \\
\hline M1 & $2,6 \mathrm{~b}$ & $47,32 \mathrm{ab}$ \\
\hline M2 & $3,2 \mathrm{ab}$ & $42,55 \mathrm{~b}$ \\
\hline M3 & $0,0 \mathrm{c}$ & $0,00 \mathrm{c}$ \\
\hline M4 & $4,1 \mathrm{a}$ & $73,50 \mathrm{ab}$ \\
\hline M5 & $4,1 \mathrm{ab}$ & $71,26 \mathrm{ab}$ \\
\hline M6 & $3,9 \mathrm{ab}$ & $82,40 \mathrm{a}$ \\
\hline M7 & $3,5 \mathrm{ab}$ & $69,36 \mathrm{ab}$ \\
\hline Média (30 dias) & 3,43 & 57,30 \\
\hline Média (60 dias) & 3,44 & 53,54 \\
\hline
\end{tabular}

Médias seguidas pela mesma letra na coluna não diferem entre si, pelo teste Tukey, a 5\% de probabilidade; M: matriz. reserva durante um período de aproximadamente dois meses após a germinação (Tiné et al., 2000). A média geral para as variáveis altura, diâmetro e sobrevivência, foram, respectivamente, aos 30 dias, $7,64 \mathrm{~cm}, 3,49 \mathrm{~mm}$ e $63,45 \%$ e, aos 60 dias, $15,69 \mathrm{~cm}$, $3,52 \mathrm{~mm}$ e $63,78 \%$. Porém valores maiores de altura nas mudas de Hymenaea courbaril Linneaus (>30,7 cm) aos 180 dias foram obtidos por Carvalho (2003) ao testar três diferentes substratos.

\subsection{Hymenaea martiana Hayne $x$ Hymenaea courbaril Linneaus}

Houve diferenças significativas entre as espécies para as variáveis germinação, altura, diâmetro e sobrevivência (Tabelas 1 e 3), sendo que a espécie Hymenaea courbaril Linneaus apresentou as maiores médias para todas as variáveis.

A espécie Hymenaea martiana Hayne apresentou variabilidade fenotípica (Teste F significativo Tabelas 1, 2, 3, 4 e 5) para todos os caracteres analisados, diferente da espécie Hymenaea courbaril Linneaus, que

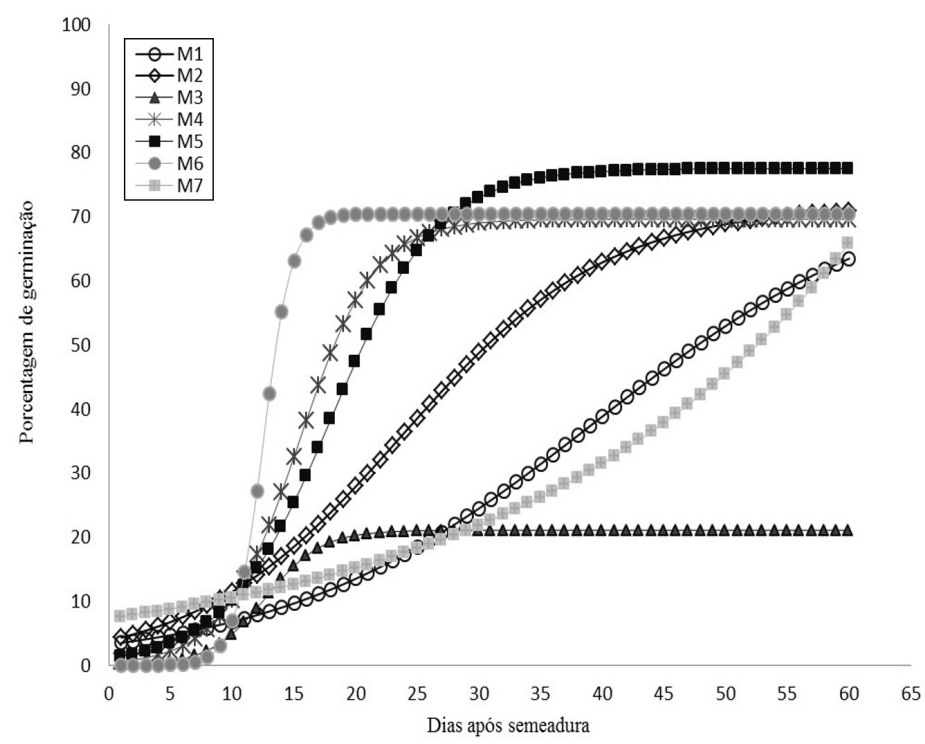

Figura 1. Porcentagem de germinação de sementes de sete matrizes de Hymenaea martiana Hayne, de acordo com o modelo $y=a /\left(1+b e^{-c x}\right)$. Diamantina-MG, novembro de 2010. M: matriz. M1_ $\hat{y}=76,0377 /(1+22,3337 e(-0,07876 x))$ $\mathrm{R}^{2}=0,96 ; \mathrm{M} 2 \_\hat{\mathrm{y}}=71,7549 /(1+17,4911 \mathrm{e}(-0,1205 \mathrm{x})) \mathrm{R}^{2}=0,86 ; \mathrm{M} 3 \_\hat{\mathrm{y}}=21,0846 /(1+276,2387 \mathrm{e}(-0,4429 \mathrm{x})) \mathrm{R}^{2}=0,98$; M4_ $\hat{y}=69,6074 /(1+152,8513 e(-0,32676 x)) \quad R^{2}=0,90 ; \quad M 5$ - $\hat{y}=77,5047 /(1+67,9155 e(-0,2335 x)) \quad R^{2}=0,96$; M6_ $\hat{y}=70,4193 /(1+5.8020,778 e(-0,3267 x)) R^{2}=0,98 ; M 7 \_\hat{y}=-3.132,6422 /(1-427,5819 e(-0,03624 x)) R^{2}=0,86$.

Figure 1. Cumulative germination curves for seven matrices of the Hymenaea martiana Hayne seeds according to the model $\mathrm{y}=\mathrm{a} /\left(1+\mathrm{be}^{-\mathrm{cx}}\right) . \mathrm{M}=$ matrices. Diamantina-MG, november 2010. M1_ $\hat{\mathrm{y}}=76.0377 /(1+22.3337 \mathrm{e}(-0.07876 \mathrm{x}))$ $\mathrm{R}^{2}=0.96 ; \quad \mathrm{M} 2 \_\hat{\mathrm{y}}=71.7549 /(1+17.4911 \mathrm{e}(-0.1205 \mathrm{x})) \quad \mathrm{R}^{2}=0.86 ; \quad \mathrm{M} 3-\hat{\mathrm{y}}=21.0846 /(1+276.2387 \mathrm{e}(-0.4429 \mathrm{x}))$ $\mathrm{R}^{2}=0.98 ; \mathrm{M} 4 \_\hat{\mathrm{y}}=69.6074 /(1+152.8513 \mathrm{e}(-0.32676 \mathrm{x})) \mathrm{R}^{2}=0.90 ; \mathrm{M} 5 \_\hat{\mathrm{y}}=77.5047 /(1+67.9155 \mathrm{e}(-0.2335 \mathrm{x})) \mathrm{R}^{2}=0.96$; M6_ $\hat{y}=70.4193 /(1+5,8020.778 \mathrm{e}(-0.3267 \mathrm{x})) \mathrm{R}^{2}=0.98 ; \mathrm{M}_{-} \hat{\mathrm{y}}=-3,132.6422 /(1-427.5819 \mathrm{e}(-0.03624 \mathrm{x})) \mathrm{R}^{2}=0.86$. 


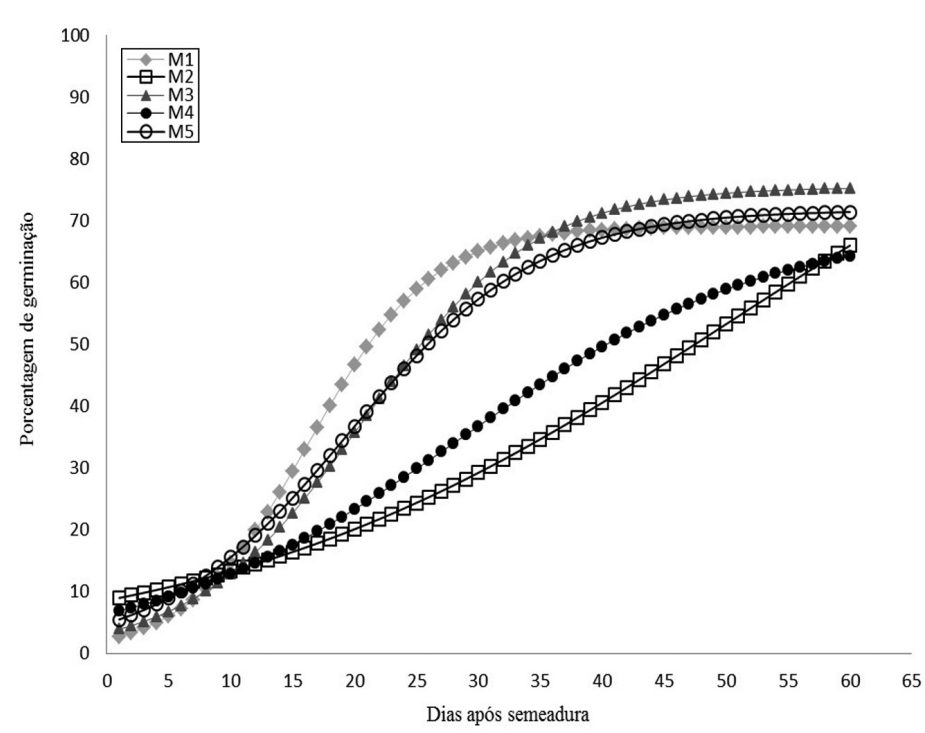

Figura 2. Porcentagem de germinação de sementes de cinco matrizes de Hymenaea courbaril Linneaus, de acordo com o modelo $\mathrm{y}=\mathrm{a} /\left(1+\mathrm{be} \mathrm{e}^{-\mathrm{cx}}\right)$. Diamantina-MG, novembro de 2010. M: matriz. M1_ $\hat{\mathrm{y}}=69,0647 /(1+28,7793 \mathrm{e}(-0,2046 \mathrm{x}))$ $\mathrm{R}^{2}=0,88 ; \mathrm{M} 2 \_\hat{\mathrm{y}}=106,7695 /(1+11,3479 \mathrm{e}(-0,0485 \mathrm{x})) \mathrm{R}^{2}=0,86 ; \mathrm{M} 3 \_\hat{\mathrm{y}}=75,4733 /(1+20,9063 \mathrm{e}(-0,1466 \mathrm{x})) \mathrm{R}^{2}=0,90$; M4_ $\hat{y}=69,4876 /(1+9,7592 e(-0,07989 x)) R^{2}=0,90 ; M 5 \_\hat{y}=71,7564 /(1+13,5877 e(-0,1330 x)) R^{2}=0,88$.

Figure 2. Cumulative germination curves for five matrices of the Hymenaea courbaril Linneaus seeds according to the model $\mathrm{y}=\mathrm{a} /\left(1+\mathrm{be} \mathrm{e}^{-\mathrm{cx}}\right) . \mathrm{M}=$ matrices. Diamantina-MG, november 2010. M1 $\hat{\mathrm{y}}=69.0647 /(1+28.7793 \mathrm{e}(-0.2046 \mathrm{x}))$ $\mathrm{R}^{2}=0.88 ; \mathrm{M} 2 \_\hat{\mathrm{y}}=106.7695 /(1+11.3479 \mathrm{e}(-0.0485 \mathrm{x})) \mathrm{R}^{2}=0.86 ; \mathrm{M} 3 \_\hat{\mathrm{y}}=75.4733 /(1+20.9063 \mathrm{e}(-0.1466 \mathrm{x})) \mathrm{R}^{2}=0.90$; M4_ $\hat{y}=69.4876 /(1+9.7592 e(-0.07989 x)) R^{2}=0.90 ; M 5 \_\hat{y}=71.7564 /(1+13.5877 e(-0.1330 x)) R^{2}=0.88$.

não apresentou variabilidade para as características altura, diâmetro do coleto e mortalidade das mudas (Tabelas 4 e 5). Essa variabilidade não encontrada na Hymenaea courbaril Linneaus pode ser explicada pela maior exploração dessa espécie, pois ela se encontra na lista de espécies medicinais brasileiras ameaçadas de extinção (IBAMA, 1992). Freitas et al. (2006) observaram que, para a espécie Myracrodruon urundeuva Allemão, o declínio do tamanho populacional e da variabilidade fenotípica e genética dessas populações é caracterizado pela expansão da população humana, devido à utilização de forma extrativista e irracional dos recursos naturais, o que pode estar ocorrendo com a Hymenaea courbaril Linneaus. A conservação dos recursos genéticos, mesmo para populações que apresentam alta taxa de variabilidade genética e estão fora da lista de espécies ameaçadas de extinção, é fundamental para as futuras gerações, no que diz respeito ao melhoramento da espécie e ao aproveitamento de genes específicos de interesse (Freitas et al., 2006).

A variabilidade encontrada para os caracteres estudados possibilita a seleção de matrizes promissoras (Melchior et al., 2006). Levando-se em consideração a produção de mudas, a espécie com maior percentual de germinação foi a Hymenaea courbaril Linneaus (Tabela 2). Além disso, possui melhor crescimento das mudas e maior sobrevivência (Tabela 4); sendo assim, a produção de mudas dessa espécie é mais viável. Porém não houve diferenças significativas entre as matrizes pelo teste de Tukey, que apresentou menor variabilidade (Tabela 2 ).

Como as matrizes selecionadas neste estudo são de ocorrência natural, não há informações sobre a idade delas, o que explica em parte a variação fenotípica entre as espécies e as matrizes. Além disso, essa variação fenotípica pode ser atribuída a outros componentes não controlados, como a condição de antropização, o solo, o clima e também pelas próprias diferenças genéticas entre os indivíduos (Ganga et al., 2010).

\section{CONCLUSÕES}

As espécies Hymenaea martiana Hayne e Hymenaea courbaril Linneaus apresentaram diferenças entre a germinação e o crescimento inicial das mudas, sendo 
a Hymenaea courbaril Linneaus a espécie com maior média para ambas variáveis

A Hymenaea courbaril Linneaus, ao contrário da Hymenaea martiana Hayne, não apresentou variabilidade fenotípica entre matrizes devido à não diferença significativa para as variáveis analisadas, o que não permite a seleção de matrizes potenciais.

\section{AGRADECIMENTOS}

Este trabalho foi apoiado pelo Conselho Nacional de Desenvolvimento Científico e Tecnológico (CNPq) e Coordenação de Aperfeiçoamento de Pessoal de Nível Superior (Capes).

\section{STATUS DA SUBMISSÃO}

Recebido: 22 nov., 2013

Aceito: 12 maio, 2015

\section{AUTOR(ES) PARA CORRESPONDÊNCIA}

\section{Priscila Fernandes de Souza}

Departamento de Engenharia Florestal, Universidade Federal dos Vales do Jequitinhonha e Mucuri - UFVJM, Rodovia MGT 367, Km 583, 5000, Alto da Jacuba, CEP 39100-000, Diamantina, MG, Brasil e-mail: souza.fernandes.p@gmail.com

\section{REFERÊNCIAS}

Andrade LA, Bruno RLA, Oliveira LSB, Silva HTF. Aspectos biométricos de frutos e sementes, grau de umidade e superação de dormência de jatobá. Acta Scientiarum. Agronomy 2010; 32(2): 293-299.

Azeredo GA, Bruno RLA, Andrade LA, Cunha AO. Germinação em sementes de espécies florestais da mata atlântica (leguminoseae) sob condições de casa de vegetação. Pesquisa Agropecuária Tropical 2003; 33(1): 11-16.

Baskin CC. Baskin JM. Seeds: ecology, biogeography, and evolution of dormancy and germination. San Diego: Academic Press; 2001.

Batista AG, Esteves EA, Dessimoni-Pinto NAV, Oliveira LG, Pires ST, Santana RC. Chemical composition of jatobá-do-cerrado (Hymenaea stigonocarpa mart.) flour and its effect on growth of rats. Alimentos e Nutrição 2011; 22(2): 173-180.
Brandão AD, Del Bem LEV, Vincentz M, Buckeridge MS. Expression pattern of four storage xyloglucan mobilizationrelated genes during seedling development of the rain forest tree Hymenaea courbaril L. Journal of Experimental Botany 2009; 60(4): 1191-1206. http://dx.doi.org/10.1093/ jxb/erp014. PMid:19221141.

Brasil. Ministério da Agricultura e Reforma Agrária. Regras para análises de sementes. Brasília: MAPA: ACS; 2009.

Busato AP, Vargas-Rechia CG, Reicher F. Xyloglucan from the leaves of Hymenaea courbaril. Phytochemistry 2001; 58(3): 525-531. http://dx.doi.org/10.1016/S00319422(01)00217-5. PMid:11557087.

Carvalho PER. Espécies arbóreas brasileiras. Colombo: Embrapa Florestas; 2003. vol. 1.

Costa RB, Gonçalves OS, Oliveira LCS, Arruda EJ, Roa RAR, Martins WJ. Variabilidade genética e estimativas de herdabilidade para o caráter germinação em matrizes de Hevea brasiliensis. Floresta e Ambiente 2005; 12(1): 74-76.

Cruz ED, Carvalho JEU. Biometria de frutos e germinação de sementes de Couratari stellata A. C. Smith (Lecythidaceae). Acta Amazonica 2003; 33(3): 381-388. http://dx.doi. org/10.1590/S0044-59672003000300004.

Cruz ED, Martins FO, Carvalho JEU. Biometria de frutos e sementes e germinação de jatobá-curuba (Hymenaea intermedia Ducke, Leguminosae - Caesalpinioideae). Revista Brasileira de Botanica 2001; 24(2): 161-165. http:// dx.doi.org/10.1590/S0100-84042001000200005.

Fernandes TT, Santos ATF, Pimenta FC. Atividade antimicrobiana das plantas Plathymenia reticulata, Hymenaea courbaril e Guazuma ulmifolia. Revista de Patologia Tropical 2005; 34(2): 113-122.

Freitas MLM, Aukar APA, Sebbenn AM, Moraes MLT, Lemos EGM. Variação genética em progênies de Myracrodruon urundeuva F.F. \& M.F. Allemão em três sistemas de cultivo. Revista Árvore 2006; 30(3): 319-329. http://dx.doi.org/10.1590/S0100-67622006000300001.

Ganga RMD, Ferreira GA, Chaves LJ, Naves RV, Nascimento JL. Caracterização de frutos e árvores de populações naturais de Hancornia speciosa Gomes do cerrado. Revista Brasileira de Fruticultura 2010; 32(1): 101-113. http:// dx.doi.org/10.1590/S0100-29452010005000019.

Gonçalves AL, Alves A Fo, Menezes H. Estudo comparativo da atividade antimicrobiana de extratos de algumas árvores nativas. Arquivos do Instituto Biologico 2005; 72(3): 353-358.

Instituto Brasileiro do Meio Ambiente e dos Recursos Renováveis - IBAMA. Plantas medicinais ameaçadas de extinção [online]. 1992. [citado em 2012 Jan 13]. Disponível em: http://www.ibama.gov.br/flora/divs/ plantasextincao.pdf.

Lorenzi H. Árvores brasileiras: manual de identificação e cultivo de plantas arbóreas nativas do Brasil. São Paulo: Planatarum; 2002. 
Machado LL, Ramos MLG, Caldas LS, Vivaldi LJ. Selection of parents and clones of mangabeira for in vitro cultivation. Pesquisa Agropecuaria Brasileira 2004; 39(5): 431-435. http://dx.doi.org/10.1590/S0100-204X2004000500004.

Maguire JD. Speed of germination-aid in selection and evaluation for seedling emergence and vigor. Crop Science 1962; 2(2): 176-177. http://dx.doi.org/10.2135/cropsci19 62.0011183X000200020033x.

Melchior SJ, Custódio CC, Marques TA, Machado NB No. Colheita e armazenamento de sementes de gabiroba (Campomanesia adamantium Camb. - Myrtaceae) e implicações na germinação. Revista Brasileira de Sementes 2006; 28(3): 141-150. http://dx.doi.org/10.1590/S010131222006000300021 .

Nogueira RT, Shepherd GJ, Laverde A Jr, Marsaioli AJ, Imamura PM. Clerodane-type diterpenes from the seed pods of Hymenaea courbaril var. stilbocarpa. Phytochemistry 2001; 58(8): 1153-1157. http://dx.doi.org/10.1016/S00319422(01)00303-X. PMid:11738399.

Rego FLH, Costa RB, Contini AZ, Moreno RGS, Rondelli KGS, Kumimoto HH. Variabilidade genética e estimativas de herdabilidade para o caráter germinação em matrizes de Albizia lebbeck. Ciência Rural 2005; 35(5): 1209-1212. http://dx.doi.org/10.1590/S0103-84782005000500037.

Rizzini CT. Árvores e madeiras uteis do Brasil: manual de dendrologia brasileira. 2. ed. São Paulo: Edgar Blucher; 2000.

Santos HP, Buckeridge MS. The role of the storage carbon of cotyledons in the establishment of seedlings of Hymenaea courbaril under different light conditions. Annals of Botany 2004; 94(6): 819-830. http://dx.doi. org/10.1093/aob/mch209. PMid:15514028.

Silva S. Árvores da Amazônia: Brasil. São Paulo: Empresa das Artes; 2006.

Souza PF, Santana RC, Fernandes JSC. Germination behaviour evaluated using the identity test For nonlinear models. Australian Journal of Basic and Applied Sciences 2013a; 7(10): 31-36.

Souza PF, Oliveira LFR, Santana RC, Fernandes ISC, Oliveira MLR, Nery MC et al. Storage of Hymenaea courbaril seeds in subzero temperature up to six months. Australian Journal of Basic and Applied Sciences 2013b; 7(13): 147-153.

Statsoft. STATISTICA: data analysis software system, version 10 [online]. 2010. [citado em 2012 Jan 13]. Disponível em: www.statsoft.com

Tiné MAS, Cortelazzo AL, Buckeridge MS. Xyloglucan mobilisation in cotyledons of developing plantlets of Hymenaea courbaril L. (Leguminosae-Caesalpinoideae). Plant Science 2000; 154(2): 117-126. http://dx.doi. org/10.1016/S0168-9452(99)00245-9. PMid:10729610.

Vargas-Rechia C, Reicher F, Sierakowski MR, Heyraud A, Driguez H, Liénart Y. Xyloglucan octasaccharide XXLGol derived from the seeds of hymenaea courbaril acts as a signaling molecule. Plant Physiology 1998; 116(3): 1013-1021. http://dx.doi.org/10.1104/pp.116.3.1013. PMid:9501133. 